\title{
Incubating a Future Metaphysics: Quantum Gravity
}

\author{
Joshua Norton
}

\section{Introduction}

In this paper, I will argue that metaphysicians ought to utilize quantum theories of gravity (QG) as incubators for a future metaphysics. In $\S 2$, I will argue why this ought to be done. In $\S 3$, I will present case studies from the history of science where physical theories have challenged both the dogmatic and speculative metaphysician. In $\S 4$, I will present two theories of QG and demonstrate the challenge they pose to certain aspects of our current metaphysics; in particular, how they challenge our understanding of the abstract-concrete distinction. The central goal of this paper is to encourage metaphysicians to look to physical theories, especially those involving cosmology such as string theory and loop quantum gravity, when doing metaphysics.

\section{Why Physics?}

There are two concerns regarding metaphysics or two errors which the metaphysician ought to avoid: dogmatism and speculative metaphysics. These are closely related errors and have received considerable attention famously by Kant in his Critique of Pure Reason and more recently by Quine (1951, 1969), Ladyman and Ross (2007, 2013), and others. The threat of metaphysical dogmatism is when philosophers assume that some particular doctrine or structure is, in fact, an item of metaphysics and that it must thereby be immune to developments in physics. The dogmatist, as I am defining him, is committed to some particular doctrine, principle, theory, or structure as being an item of pure metaphysics (see (A) below). I will define pure metaphysics to include all propositions, theories, principles, etcetera, whose truth value is independent of the truth of any physical fact. In order to bring this usage in line with traditional accounts, we will excise from this set of truths any truths which are mathematical or logical in nature. According to the doctrine as defined 
so far, if some principle or doctrine is thought to live safely on the side of pure metaphysics, then the principle is, by definition, immune to developments in our physical theories: no matter how the church bells chime, Leibniz's principle of the identity of indiscernibles (PII) stands, for instance. We will come back to the PII in a later section. The error of dogmatism is not found in believing that there are items of pure metaphysics but in the believing with certainty that some particular doctrine is amongst them. In the following, I will not argue against pure metaphysics as such, but against dogmatic attitudes towards it.

Addressing reckless or speculative metaphysics, Kant claims:

And precisely in these latter cognitions, which go beyond the world of the senses, where experience can give neither guidance nor correction, lie the investigations of our reason that we hold to be far more preeminent in their importance and sublime in their final aim than everything that the understanding can learn in the field of appearances, in which we would rather venture everything, even at the risk of erring, than give up such important investigations because of any sort of reservation or from contempt and indifference. These unavoidable problems of pure reason itself are God, freedom and immortality. But the science whose final aim in all its preparations is directed properly only to the solution of these problems is called metaphysics, whose procedure is in the beginning dogmatic $\cdots$

Furthermore, if one is beyond the circle of experience, then one is sure of not being refuted through experience. The charm in expanding one's cognitions is so great that one can be stopped in one's progress only by bumping into a clear contradiction. (1998 p.139, 140)

The worry here is that when constructing metaphysics we err in thinking it sufficient for knowledge that our system be consistent. The psychological effect of a highly complex system of doctrines which also happens to be consistent is powerful. Couple consistency with a modicum of elegance, and history has shown how the human psychology is overwrought with a compulsion to affirm the absolute necessity of our metaphysics. The problem, of course, is that there are infinitely many systems of comparable complexity and elegance which are each internally consistent and yet pairwise inconsistent. If any two metaphysical systems are inconsistent, these systems describe incompatible ways that the world might be. Since incompatible systems cannot both be true, consistency is not sufficient for truth. This result holds for contexts other than metaphysics. There are also infinitely many physical theories 
which are internally consistent but pairwise inconsistent. In the case of physical theories, we have the help of a further constraint. We require not only that our physical theories be internally consistent, but also that they be empirically adequate. ${ }^{1}$ Though for those who are realists about scientific theories, this additional constraint does not solve all of their problems. There are always multiple interpretations of any theory, each internally consistent and empirically adequate and yet mutually incompatible. $^{2}$ In summary, the error of speculativism is to ignore physical truths, relying on consistency alone, in building a metaphysics. The error of dogmatism is to ignore physical truths when adhering to a metaphysics.

Before continuing, allow me to discharge a task which I have no intention of undertaking. In the following, I will not attempt to adjudicate what constitutes the difference between a physical theory, principle, fact or structure, versus a metaphysical theory, principle, fact, or structure. Nor will I argue for a particular relation between pure metaphysics, as defined here, and metaphysics (see (A) through (C) below). Perhaps these are the same concept and perhaps not. The terms 'physical' and 'metaphysical' are notoriously vague and have changed in meaning since Aristotle's time. Aristotle does not use the word 'metaphysics' himself though he does identify a set of questions which are different from those addressed in his texts on physics. These latter questions have since been labeled as 'metaphysics' and questions, in addition to Aristotle's, have been added to them. The argument I will make holds no matter what the actual difference is between physics and metaphysics. This argument can be made even if the truths of metaphysics and physics are independent since my argument will depend only on claims regarding what humans can reasonably count as knowledge. In order to streamline the following discussion, unless otherwise noted, I will speak as though there is a difference in kind between physical and metaphysical structures and our theories about them.

Dogmatism and conceptual recklessness are not incompatible faults. Indeed, it seems that the more brazen the offender of either error the more likely he will also run afoul of the other. The more the metaphysician is wooed by the internal consistency and elegance of his metaphysics, the more likely he will take this metaphysics as being not merely true but essential. ${ }^{3}$ Granting these dangers, the reader might well wonder, "what has physics got to do with it?" I will provide three alternatives

1 For 'empirical adequacy' see van Frassen (1980). Alternative concepts might do equally well.

2 One could always append to any theory a structure which happens to be empirically relevant only in regimes or energy scales beyond that which we can currently engage. This, of course, is nothing more than the infamous problem of underdetermination of theory by data.

3 This concern applies to physical theories as well. Examples of this can be found in the more excited pronouncements from the string theory community. For why I say 'essential' see the following footnote. 
for what we might take the relation between physics and metaphysics to be; once that task is done, I will address this question. To reiterate, I will not argue for the truth of any of these alternatives. I mention them only to argue that no matter one's understanding of the relation between physics and metaphysics, one would be mistaken to not assess their metaphysics in light of physics.

(A). By definition, physics and metaphysics govern non-overlapping domains. The truth or falsity of some metaphysical thesis is independent of the truth or falsity of any physical thesis. Or, less strongly, the truth of at least some metaphysical theses are independent of which physical theories are true of the world. In the following I will use ' $(A)$ ' to refer to the stronger thesis which I have called 'pure metaphysics.'4 Besides the condition of independence, I have not attempted to provide an account for what makes something purely metaphysical or physical. One may wonder what intrinsic properties must a thing have to be a physical thing and likewise, a purely metaphysical thing? I will leave this question for the proponents of $(\mathrm{A})$ and will not attempt to answer it. For the sake of my project, all I need to distinguish (A) from the following accounts is the independence condition (see $(\mathrm{B}))$ and the commitment to some metaphysics (see $(\mathrm{C})$ ).

(B). According to Quine, there is no intrinsic difference between physical and metaphysical theories or the structures to which they refer. Famously, Quine claims that "no statement is immune to revision" be that a statement of the natural world or of logic (1951, p.40). Quine's point is that there are no theses about the world which are safe from revision in light of recalcitrant empirical evidence. The difference between physics and metaphysics is a matter of degree and is captured in the structure of our web of concepts. Those concepts which are easier, according to some internal criterion, to modify in light of recalcitrant experiences are labeled 'physical'. Whereas, those statements whose revision would cause widespread secondary modifications throughout the web, such as mathematics and logic, live closer towards the

4 Any doctrine meeting the demands of $(\mathrm{A})$ is not merely metaphysical but necessarily so. If $\mathcal{D}$ is a pure metaphysical doctrine in the actual world, then its truth value is unaffected by which physical laws $(\mathcal{L})$ are true of the actual world. In other words, $\mathcal{D}$ is consistent with any $\mathcal{L}$. If this is so, then for all $\mathcal{L}$ there exists some possible world $\left(\mathcal{P}_{\mathcal{L}}\right)$ accessible to the actual world in which both $\mathcal{L}$ and $\mathcal{D}$ are true. I will prove that $\mathcal{D}$ is necessarily metaphysical-in the sense of (A)-by showing that in each $\mathcal{P}_{\mathcal{L}}, \mathcal{D}$ is consistent with each $\mathcal{L}^{*}$. If this were not so, then there is some $\mathcal{L}^{*}$ such that $\mathcal{P}_{\mathcal{L}^{*}}$ is inaccessible from $\mathcal{P}_{\mathcal{L}}$. However, if the accessibility relations between possible worlds is both reflexive and transitive (i.e Euclidean), then this is false. In conclusion, if the modal access relations between possible worlds is at least symmetric and transitive, then were $\mathcal{D}$ an item of pure metaphysics, $\mathcal{D}$ would be so necessarily. 
center of the web (p.41). Some of these more central statements or doctrines we call 'metaphysics.' According to the Quinian, there is no system-independent way of managing one's conceptual web and therefore no system independent way of carving the world into physical and metaphysical structures. Notably, there are no items of pure metaphysics according to the Quinian.

(C). According to the positivists, all that can be affirmed about the world is that which is true a priori or expressed in empirically verifiable sentences. According to A.J Ayer,

It should be mentioned here that the fact that the utterances of the metaphysician are nonsensical does not follow simply from the fact that they are devoid of factual content. It follows from that fact, together with the fact that they are not a priori propositions (1936, p.41).

Ayer requires that we ask of every sentence:

Would any observations be relevant to the determination of its truth or falsehood? And it is only if a negative answer is given to this second question that we conclude that the statement under consideration is nonsensical (p.38). ${ }^{5}$

Accordingly, pure metaphysics is a meaningless enterprise. The future of philosophy, according to the positivists, is the elucidation of our physical theories and the ontology suggested by them. Given the quote from page 41, it seems that according to Ayer, metaphysics simpliciter is to be identified with pure metaphysics, and not also with the expression of analytic truths. Consequently, in referring to (C) I will often drop the prefix 'pure'. In describing the positivist, project I have focused only on Ayer's radical form of it. To be sure, there are positivists of a more tolerant variety, such as Carnap, though for the purposes of this paper I will not intend anything other than Ayer's positivism when referring to $(\mathrm{C})$.

Positions (A) - (C) do not exhaust the space of possibilities; ${ }^{6}$ however, they do provide a broad platform from which to address my claim that we ought to use quantum theories of gravity as incubators for a future metaphysics. My thesis is that theoretical physics and, in particular, quantum theories of gravity are well poised to help us in probing both the physical and metaphysical structures of reality.

5 Where, presumably, the sentence under consideration is not one of mathematics or otherwise known a priori; cf. the previous quote which comes later in his text.

6 For instance, neither Aristotle nor Kant fall naturally into any of these categories. 
Quinians and positivists will, in principle, raise no objections against the use of quantum theories of gravity in affecting a new metaphysics. Two small concerns should be addressed, however. First, one might wonder why quantum theories of gravity are specially important to our metaphysics. Surely, both the positivists and Quinians hold all of science important for metaphysics. While Quine certainly took all of science to be important for our conceptual web, I will argue that the conceptual revolution(s) suggested by both string theory (ST) and loop quantum gravity (LQG) are of a particularly deep kind. My thesis is not that only QG is relevant for our understanding of the metaphysical structure of reality but that QG is especially wellsuited for doing so. Second, the positivists do not think that there is a meaningful area of study called metaphysics. Granting this objection, my thesis is rather: we ought to use quantum theories of gravity as incubators for whatever metaphysics there is. This condition is trivially satisfied for positivists.

Given the nature of their position, Quinians are predisposed against, though not immune from, the errors of dogmatism and speculative metaphysics. To illustrate this, consider option (B). If one accepts that metaphysical and physical facts are of one kind, conceptually wed within a coherent whole, then, by definition, one must pay attention to which physical theories are 'true' in deciding which metaphysical theories are 'true'. 7 According to the Quinian, a change in any part of the web affects some other parts. A drastic enough change in what we take the physical world to be like might even require a retooling of more central parts of our conceptual web. If general relativity (GR) is replaced by a quantum theory of gravity, this might rattle the web enough to affect our metaphysics $(\S 4.3)$.

While it might come as no surprise that those in camps (B) and (C) will accept the thesis of this paper, so too should those in camp (A). The reason for this is that even if pure metaphysics were possible, we do not know when we are doing it. Assuming that there is a set of pure metaphysical facts does not mean that we have correctly identified them. It is possible to be both an adherent of (A) and yet not be dogmatic. Position (A) says that there are pure metaphysical facts and that these facts hold independent of whatever else is true physically. The dogmatist adds the further commitment that some particular structures are in fact items of pure metaphysics and therefore immune from the revolutions in physics. What I am advising here is that the adherents of (A) be agnostic about which facts are metaphysical and to use physics as a means of purifying the metaphysics which they think they have. In the following I will describe historical episodes where we had thought some thesis or principle to be of pure metaphysics and about which we were

\footnotetext{
7 I have placed 'true' in scare quotes to signal that in the context of conceptual-webs, the proponent, presumably, endorses a deflated notion true: truth-qua-coherence.
} 
mistaken. These historical cases argue against both dogmatism as well as speculative metaphysics and in favor of an agnostic version of (A).

Note, it is unlikely that any of us avoid all instances of dogmatism. In choosing some position $(\mathrm{A})-(\mathrm{C})$ we take a stand on how the world is. We are not forced to adopt the following attitude, but if the reader is anything like this author, then we think our choice of $(\mathrm{A})-(\mathrm{C})$ is true independent of what physical facts are true. For instance, in saying that $(\mathrm{B})$ is true, I am making a claim about how the set of physical structures is related to the set of metaphysical structures and my commitment is independent of which structures happen to occupy those sets. Since this relation is independent of which items compose the set of physical truths, my belief in or commitment to (B) is unaffected by the actual physical structure of the world. No matter what physics reveals to me, $(\mathrm{B})$ is true and I will organize the world according to it. This of course is the essence of the dogmatic attitude defined above. Dogmatism, in its varied forms, is ubiquitous but often difficult to identify.

\section{Conceptual Revolutions}

In this section, I will consider four cases from the history of science in which a metaphysical, or putatively metaphysical, thesis foundered upon some development in physics and was subsequently modified. These examples demonstrate both the error of being dogmatic about the necessity of our metaphysics, and how physics can be used to guide the construction of metaphysical systems. Before beginning, a brief caveat is in order: each of the following examples involve textual nuances and, in some cases, interpretive uncertainty regarding the physics. I will present what I take to be plain or "straightforward" readings of philosophical texts as well as the relevant physics but admit that each of the following conflicts might be avoided through a modicum of philosophical or hermeneutical gymnastics. At the very least, one could always deny that it is appropriate to interpret our scientific theories realistically. If our scientific theories are appropriately understood in only an instrumental fashion, then many of the following conflicts dissipate.

Example 1: Aristotle, on the first cause and its effect. For Aristotle, it was a metaphysical fact that there is a prime mover who is responsible for causing the eternal circular motion of the heavenly bodies.

There is, then, something which is always moved with an unceasing motion, which is motion in a circle; and this is plain not in theory only but in 
fact. Therefore the first heaven must be eternal. There is therefore also something which moves it. And since that which moves and is moved is intermediate, there is something which moves without being moved, being eternal, substance, and actuality.

The final cause, then, produces motion ... motion in space is the first of the kinds of change, and motion in a circle the first kind of spatial motion; and this the first mover produces. The first mover, then, exists of necessity... (Met. XII, 7)

The conviction that there is a first mover which eternally causes heavenly bodies to follow circular orbits, ruled Astronomy for roughly fifteen hundred years until Kepler postulated elliptical orbits in $1605 .^{8}$ All other logically possible trajectories for the planets were deemed impossible given prior Aristotelian commitments. Our dogmatism over the the nature of the heavens hamstrung astronomers' ability to imagine the world being otherwise. ${ }^{9}$ The revolution ushered in by Copernicus et.al was not merely a revolution of astronomy but in general cosmology and metaphysics as well. According to Newtonian and now Einsteinian cosmology, whatever the nature of a prime mover, such a mover is not the cause of eternal circular orbits. We now believe that the heavens began to exit, and that no heavenly body has a circular orbit about the center of the universe. According to modern cosmology the universe does not have a center, and what orbits are there, are never perfectly circular (due to perturbing factors of other bodies). In moving away from Aristotelian cosmology, the age and general dynamics of the heavenly bodies shifted from being questions of metaphysics to those of empirical investigation. ${ }^{10}$ What Aristotle held to be true for philosophical reasons, we now reject on empirical grounds.

Example 2: QM and the PII. The principle of the identity of indiscernibles (PII) is claimed by Leibniz to be something like a law of metaphysics. According to the PII, "it is not true that two substances may be exactly alike and differ only numerically"

8 Aristotle's cosmology was formed sometime in the third century BC but got its most developed early treatment in Ptolemy's Algamest (100-170 AD). Ptolemy was not a strict Aristotelian and deviated from Aristotle's crystalline-shell structure significantly.

9 There are some outliers in this regard: Leucippus and Democritus 5th century B.C., Pythagoras and Herclides 4th, and notably, Aristarchus who, in the 3rd century B.C., had a heliocentric model similar to Copernicus' (Kuhn, 1957/2003).

10 This claim involves only the general dynamics of heavenly bodies such as the shape of the planetary orbits and does not include some particular features of these orbits such as their respective tilt or their orbital speeds. These latter features are physical hypothesis according to Aristotle to be discovered empirically. 
(Leibniz, 1902, IX). The truth of the PII is supposed to be independent of the actual physical goings-on in the world. Yet, the PII is argued to fail in light of quantum mechanics (QM). While philosophers debate the merit or the proper form of this conflict, ${ }^{11}$ on the face of things, QM contradicts the PII. According to QM, it is possible to form systems of multiple particles which share all their monadic properties: mass, charge, spin, position etcetera. The quantum state of this system is such that whatever can be predicated of any one particle can be predicated of the other. Prima facia then, QM allows non-discernible, non-identical substances.

The current example putatively illustrates that what was taken to be a principle of metaphysics (PII) has come into sharp conflict with physics. Who could have foreseen the invention of quantum mechanics and predicted this conflict? In general, we cannot predict the extent or depth to which an emerging theory of physics might require a retooling of our conception of reality. Importantly, that retooling might suggest that we modify some principle of metaphysics. For those who want to allow for the possibility of pure metaphysics, examples such as this should teach us not to be dogmatic about what we currently conceive of as pure metaphysics. ${ }^{12}$

Allow me a moment to re-emphasize that every one of the four cases here considered are not without their controversy. Each of these cases has inspired a healthy query into foundational issues in physics and metaphysics where some authors have attempted to ameliorate the putative conflict between physics and metaphysics. My intention is not to chose a side in the debates which have sprung up but merely to present the canonical accounts of the relevant conflicts. From this point forward, I will take it as read that the issues being discussed are more subtle than can be presented. Indeed, an exploration of the subtleties in these cases is besides the point. My argument requires only that there have been conflicts between our physical and metaphysical theories, not that some favored resolutions rise from the ashes of these conflicts.

Example 3: Kantian Space. According to Kant, space is a form of our intuition modeled by Euclid's axioms. In his "Inaugural Dissertation of 1770" Kant claims:

The concept of space, therefore, is a pure intuition... the fundamental

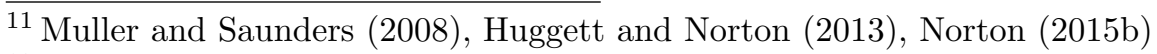

${ }^{12}$ Quantum mechanics threatens not merely the PII, but what we take to be the logical structure of reality. Given most standard interpretations of QM, i.e. not including hidden variable interpretations, not all statements of the form $P \vee \neg P$ are true. For instance, when a particle is in an eigen-state of momentum, the particle is described as somehow being located everywhere and yet nowhere. For such a particle, it is neither true of the particle that it is 'here' nor is it not true that it is 'not here'. See, Birkhoff and von Neumann (1936).
} 
form of all external sensation. This pure intuition is in fact easily perceived in geometrical axioms.

Since, therefore, nothing at all can be given the senses except conformably to the primary axioms of space and their consequences which are taught by geometry... (1894, p64-67).

For Kant the axioms or structure of space are the axioms or structure of Euclidean geometry. ${ }^{13}$ Laying aside whatever textual controversies there are, ${ }^{14}$ and taking this text at face value, Kant is committed to the essentiality of Euclidean geometry. This commitment gets Kant into trouble with the discovery of non-Euclidean geometry and the theory of general relativity which makes use of it. The geometry of space, according to GR, is dynamic and does not have to obey Euclid's axioms. For example, according to Kant (applying Euclid), "between two points [of space] there is but one straight line" (p.64) which need not be true in GR. In GR, it is possible for infinitely many straight lines to pass through two points. The often cited example of this is the North and South pole, and the lines of longitude which pass through them. The lines of longitude look curved when we draw them but are straight according to the theory of GR. ${ }^{15}$

Similarly, if string theory (ST) is true, space has somewhere between 10 and 26 dimensions (most of which are rolled up into tiny loops) and yet according to Kant "in space there are no more than three dimensions" (p.64). Both Kant and Newton took space to be three-dimensional and Euclidean and, therefore, both are wrong given GR and doubly wrong if ST turns out to be true. Though they are both in error, Kant's error is of a different kind than Newton's. Unlike Newton, Kant's theory of space is not a physical theory discoverable through empirical investigation:

We are concerned solely with this. Space and time are its pure forms, sensation in general its matter. We can cognize only the former a priori, i.e., prior to all actual perception, and they are therefore called pure intuition... . (1998 p.185)

According to Kant, our understanding of space is a priori and independent of our experiences. Given its status as an item of pure knowledge, Kant's theory of space

\footnotetext{
${ }^{13}$ Noting that non-Euclidean geometry was still to be discovered.

${ }^{14}$ Like most aspects of Kant scholarship what Kant means by the above statements is debated (Smith 2003, p.117)

${ }^{15}$ To be clear, this example is intended only as an analogy. Taken literally it is not true that GR describes longitudinal lines as being straight. The example is meant to provide a picture for how more than one straight line can intersect the same pair of distinct points.
} 
is a metaphysical theory (of the acceptable, non-speculative kind). If knowledge of the nature of space is independent of experience, then no empirical theory can contradict it. However, as we have seen, GR does contradict it. This conflict should not be possible if space is known a priori. Here again, we see an example where some putatively metaphysical thesis is shown to be false by a hitherto unimaginable physical theory.

Example 4: Future contingents. It is a common piece of our background beliefs that existence is attached to the present and occurs moment by moment. According to this pre-theoretic metaphysics, the past is no longer and the future is yet to be. As I write this, I exist and so does this computer but Aristotle, and my youth no longer exist, and the events of tomorrow are yet to exist. I will refer to this assumption as "presentism". ${ }^{16}$ Because we take existence to be attached only to the present, there is a question for how to assign truth values to statements about future contingents. The puzzle over future contingents goes back at least as far as Aristotle. Aristotle asks us to consider the following propositions: (a) 'there will be a sea battle tomorrow' and (b) 'there will not be a sea battle tomorrow' (Int. I, 9). We, and he, tend to think that one of these mutually exclusive and jointly exhaustive statements is true. Yet, if so, on what grounds would either be true? Presumably if 'there will be a sea battle tomorrow' is true, it is true in virtue of there being a sea battle tomorrow. Truth makers, today, for statements about the future seem to be determinant, existent structures in the future. If this is so, then, how can presentism be true? How can existence be limited to the present if there are existent future structures?

A solution to this puzzle requires, in part, that we stipulate some particular relationship between language, truth, and reality. For instance the logician Jan Łukasiewicz, in the early twentieth century, proposed that statements of future contingents need not obey the law of the excluded middle. In formalizing this logic, Łukasiewicz (1970) introduced a third truth value for future contingents. According to Eukasiewicz, statements of the kind 'there will be a sea battle tomorrow' are neither true nor false but undetermined. The point of this example is to highlight how a prior commitment to the nature of existence - as being attached to the present - trickles out and affects a wide swath of our remaining metaphysics. If we take existence to be attached only to the present then the logical structure of the world might fail to include determinant truth values for statements about the future.

One need not modify the logical structure of the world in order to make sense of future contingents. Why, for instance, assume that existence is tied up with the

\footnotetext{
${ }^{16}$ Not to be confused with the presentist who holds additional commitments such as the passage of time.
} 
present? This commitment, for many of us, is an accident: we have unreflectively assumed that this is what it means to exist. What else could exist than that which exists now? According to the special and general theories of relativity, existence is not tied to the present but distributed equally to all moments of time. These theories do not allow the present to be used as tool for carving reality into objects or events which objectively belong to the past and those which objectively belong to future. ${ }^{17}$

According to special relativity, an event $\alpha$ in our past is related to our past in the same way that a cat on our right, is related to our right. ${ }^{18}$ Just as a different traveller (observer) might judge the cat as being on her left, so too she might judge $\alpha$ as being in her present. This is one of the surprising modifications suggested by the theories of relativity. In order for a statement about the past or future to be true, according to relativity, one needs to specify the observer, relative to whom, the statement is being made. Because of the relativization of 'past', 'present,' and 'future', we can no longer, innocently, attach existence to our present moment of time. If GR is true our metaphysical bias is false: that which exists is not the same as that which exists now.

In each of the aforementioned cases, some aspect of the word was once thought to be metaphysical, a product of pure reason, or merely outside the ken of empirical investigations and we were wrong. In some of these examples, the putatively metaphysical doctrine was explicitly formulated as being an item of metaphysics (Examples 1-3) and yet, as Example 4 illustrates, sometimes we hold metaphysical prejudices unintentionally. These aforementioned cases, and the ones to follow (§4.3), ought to temper our dogmatic tendencies, if there be any. These examples demonstrate that we cannot expect to predict how drastically our future physical theories might reshape our current perception of reality. While in the grip of the Ptolemaic-Aristotelian framework, one could not foresee the invention of the concept of inertia which underpinned the Copernican revolution and allowed the Earth to spin. So also today, we cannot foresee what new concepts will be required by our future physics and thereby cannot foresee which "impossible" things will become natural or obvious.

The claim here is not that once physics steps in, any interpretive or conceptual issues are solved. The claim is not that physics automatically decides how we must understand reality, but rather, that physics opens up possibilities of which we were not previously aware and in some cases mitigates against options we had once thought attractive. It is in this way that physics can help guide an alternative metaphysics. If

\footnotetext{
${ }_{17}$ Or, in the case that the future is open, those which possibly belong to the future.

${ }^{18}$ In order for this to work, $\alpha$ has to lie outside our past light cone. Such an event is in our past though, admittedly, not in our causal past.
} 
one is a Quinian about metaphysics then the guidance of physics is straightforward: one ought to modify the more central aspects of their web in order to be consistent with their favored interpretation of the new physics. If one adheres to option (A) and aims to construct a pure metaphysics, then in each of the above cases, our new physical theory instructs the metaphysician how to further purify her account. For instance, with respect to Example 1, the pure metaphysician might heed the advice of Einstein and excise from her metaphysics any claims regarding the age or essential shape of celestial orbits.

\section{Quantum Gravity}

In the following, I will demonstrate how quantum gravity places pressure on some of our current metaphysics. I will first outline the theories of LQG and ST and then examine five formulations of the abstract-concrete distinction showing how each of these formulations fails to hold if LQG or ST is true. If either theory is true, then there is no abstract-concrete distinction or we need a new formulation of it. The lesson I will draw from this is that philosophers ought to use quantum theories of gravity in order to test the necessity of their favored metaphysics and to guide their building of new alternative systems. The target audiences for this paper are general philosophers and philosophers of science and not primarily philosophers of physics, though I hope there is something for everyone. As a result, I will not provide a detailed account of the inner workings of either LQG or ST. The following two subsections are, what I hope to be, faithful gleanings of these theories, relevant for the current discussion. Anything like a mature presentation of either theory would require many more pages and much more mathematics than is warranted for our purposes. For more complete presentations of these theories, see the cited works.

\subsection{LQG}

The theory of LQG aims to provide a theory of quantum gravity or, equivalently, quantum geometry. Geometry in general relativity is modeled by the mathematical metric field $G .^{19}$ This field encodes all the geometric properties which we associate with the relativistic spacetime of GR. Spatial lengths, areas, volumes, and temporal duration are all encoded by the field $G$. Physical properties such as momentum, force, and energy are also modeled, at least in part, by the metric field. The momentum of a car, for instance, is defined as $m \frac{d x}{d t}$ where $d x$ and $d t$ are infinitesimal, spatial,

\footnotetext{
${ }^{19}$ For a general account of the theory see (Rovelli, 2004).
} 
and temporal lengths. A similar analysis applies to force and energy. In providing a quantum description of geometry, LQG tosses out the metric field in favor of quantum states which model what might be thought of as quantum geometric structures. However, in so doing, the models of LQG describe a fundamental physical reality which lacks lengths, areas, and volumes as standardly conceived and as we normally experience. These models also fail to include many of our basic physical structures such as momenta, force, and energy. ${ }^{20}$ While our standard, classical structures are missing from the physics of LQG, there are other structures, quantum gravitational or quantum geometrical structures in their place. Its not as though we don't have anything in LQG, we just have very unfamiliar things.

For the sake of this, all too brief, introduction, I will refer to the fundamental ontology of LQG by the name 'spin-networks'. These networks represent the quantum geometric properties of the world. If one takes spatial geometry to be a constitutive structure of spacetime then, strictly speaking, spacetime, as standardly understood, is not a fundamental aspect of reality. However, the quantum geometry of LQG looks like classical geometry at low energies when spin-networks take the form of "weavestates" (what weave-states are, is not important). In these special cases, the theory describes structures which correspond, roughly, to the geometry of our familiar experiences. Because of these features, Huggett and Wüthrich (2013) and Oriti (2014) claim that spacetime is emergent from the spin-networks of LQG. I will follow the convention of the literature and will continue to say "spacetime emergence" though, in the case of LQG, it might be more accurate to say "space emergence" given that the weave-states only encode the geometry of the three-dimensional hyper-surfaces of spacetime.

Though the preceding outline of LQG was rather brisk, many of the remaining details will not be important for our purposes. What is important is the claim that spacetime is not a fundamental piece of reality according to the received interpretation of the theory. One very reasonable worry is that a world of physical objects which does not include spacetime is a world that is hard to imagine or comprehend. This is exactly the reason why quantum theories of gravity are useful for putting pressure on our background metaphysics. For a fuller discussion of the theory of LQG from a philosophical perspective see Baker (2016), Norton (2015a), Wüthrich (2014), Lam and Esfeld (2013), Rickles (2005, 2006), Pooley (2006), Earman (2002), Maudlin (2002), Earman and Belot (2001). To be fair, there are ways of interpreting

\footnotetext{
${ }^{20}$ Presumably, in the new theory, we will have to update what we take momenta, force, and energy to be. Some of our old concepts will likely be revised and carried over, while other concepts might be abandoned altogether. It is also likely that novel physical concepts will play a role in the new theory which we did not need in the old.
} 
the theory such that spacetime does not disappear fundamentally (Norton 2015a), though these are not the received interpretation according to the literature.

Going forward, when I claim that spacetime is not fundamental, what I mean is that, as defined in GR, there are good reasons for thinking that spacetime is not represented by the mathematics of LQG or ST. However, as mentioned above, the quantum states of LQG are able to reproduce or mimic the geometry of GR (in special cases). In this way, spacetime-qua the geometry of GR-is an effective structure (see Huggett and Wüthrich (2013)). If one interprets effective structures as having an existence all their own, over and above fundamental reality, then one might claim spacetime to be an emergent piece of ontology. Since I do not want to implicitly endorse an interpretation of effective structure, in this paper I will claim the weaker thesis that spacetime fails to be fundamental rather than claiming it fails to exist en tout.

\subsection{String Theory}

Unlike LQG, string theory was not formulated as a quantum theory of gravity, but it turns out that ST does this for free, so to speak.

There are a series of papers ${ }^{21}$ which together suggest that the physical space of our phenomenological experiences is not the physical "space" fundamental to string theory. If this conclusion is true then, were ST true, spacetime as we know it is a secondary, higher-level phenomenon. According to some authors, Huggett and Wüthrich (2013), spacetime is emergent from a more fundamental reality described by string theory. I will not explain the details of these arguments but merely report on what is argued to be the case. What concerns us is not why exactly spacetime fails to be modeled in ST, but that it does fail to be modeled and how this affects our metaphysics of the world.

In "Target Space $\neq$ Space" Huggett exploits the T-duality of ST in order to argue that the physical space of ST lacks definite size. According to T-duality, ST is consistent with the size of space being either very tiny or very large. According to this argument, ST has indeterminate spatial size unlike phenomenological space which is large. Huggett uses this fact to argue that the physical space of ST is not the phenomenological space of our daily experience. The space of ST, according to Huggett, is some less structured portion of reality. Other authors (Rickles (2013.a/b) and Teh (2013)) exploit mirror symmetry and what is known as the AdS/CFT correspondence to argue that the space of ST lacks certain global topological features

${ }^{21}$ Huggett and Wüthrich (2013), Huggett (forthcoming), Matsubara (2013), Rickles (2011), Dawid (2007). 
which we normally associate with phenomenological space.

Mirror symmetry is used by Rickles to argue that in an important sense, the "shape" of the physical space of string theory is indeterminate while the AdS/CFT correspondance is used to argue that the dimensionality of this space is indeterminate. The inference made by these authors is the same as Huggett's: the physical space which shows up in ST is not phenomenological space of spacetime but something less structured. Lastly, Huggett and Vistarini (2015) explain how ST captures the geometric structure of spacetime but only at the low energy limit. This means that when probing phenomenological space at high energy, geometry as we know it, ceases to exist. More precisely, geometry does not exist, as such, but rather some stringy vibrations exist and these produce phenomena which, at low energies, we experience geometrically. According to this view and mirroring the results of LQG, geometry is not a fundamental structure of reality. If one takes spatial geometry to be a constitutive structure of spacetime, then spacetime is not fundamental.

In the following, I will make claims regarding QG but admit that I am only warranted in making claims regarding ST and LQG. There are other versions of QG, causal set theory, shape dynamics, and others, which I do not consider.

\subsection{QG and Abstract Objects}

Because of the lack of spatiotemporal structure (lengths, areas, energy), some ${ }^{22}$ have argued that spacetime is fundamentally absent in QG. And yet, if spacetime fails to be a fundamental, some of our metaphysics will need to be altered. In the following, I will analyze five accounts of the abstract-concrete distinction, and show how each of these distinctions fails to hold if QG is true. ${ }^{23}$ In short, if QG qua either ST or LQG, as described by its received interpretation, is true, then there is no distinction between fish and the number $\pi$, at least with respect to their concreteness. ${ }^{24}$ There are at least three responses to this curiosity. Firstly, we can accept the conclusion. For those like Maddy (1990) who independently argue that some mathematical objects are concrete, or Ladyman and Ross $(2007, \S 3.6)$ who have their own reasons for denying the distinction, this result from QG might be welcomed. Secondly, we might infer that since fish and $\pi$ are not the same kinds of things, the received interpretations of QG must be false. Or, thirdly, we can interpret the results of QG as requiring

\footnotetext{
${ }^{22}$ Huggett and Wüthrich (2013), Oriti (2014).

${ }^{23}$ Others have also discussed the relation between abstract and concrete objects and LQG (Lam and Esfeld 2013, Lam 2015, Norton 2015a).

${ }^{24}$ By 'received' I do not mean to suggest that the entire community explicitly endorses these interpretations. But rather, that the general thrust or spirit of the interpretations which are endorsed in the literature is in agreement with the interpretations presented as 'received'.
} 
a new account of abstract objects. While it is unlikely that there is a general answer for how we ought to respond to conflicts of this kind, it would be a mistake to throw out QG, or rather, our interpretations of it, without first analyzing how committed we are to the old formulations of the distinction. To these formulations, I will now turn.

Case 1: A standard and ubiquitous account for what distinguishes abstract and concrete objects grounds the distinction in how these objects relate to space or spacetime. According to this proposal, an object is concrete if and only if it is spatiotemporally located, or locate-able. This latter clause is to allow quantum particles in spatial superpositions to count as concrete. If we take spacetime to be that structure which distinguishes concrete objects from abstract objects, then the abstract-concrete distinction collapses in QG since there is no spacetime according to the theory. One route we could take to amend this approach is to upgrade the conditions of the distinction in terms of the quantum spacetime of LQG or in terms of the non-phenomenological space of ST.

Both LQG and ST include mathematical structures which can be interpreted as being akin to something like spacetime though not spacetime itself. For example, in the case of LQG, if one is a relationalist with respect to spacetime in the sense that all there is to spacetime are the spatiotemporal relations encoded in the metric field of GR, then one might simply switch their allegiance from the classical spacetime represented by the metric field of GR to what we will call the "quantum spacetime" defined by the quantum geometric relations of LQG (for other options see Norton (2015a)). Once this allegiance is switched, we could define the distinction between abstract and concrete objects in terms of it: an object is concrete if and only if it is in quantum spacetime. Or equivalently, an object is concrete if and only if it stands in quantum geometric relations, i.e. if it couples to the spin-networks of LQG.

Granted, the standard spatial-account of the abstract-concrete distinction runs into trouble for a variety of reasons independently of QG. As I have just alluded to and as we saw in $\S 3$, particles in a superposition of spatial position do not exist at any place in space. However, these particles are locate-able since it is possible to force quantum particles to occupy some spatial location which, of course, is not true for the number $\pi$. Things are a bit worse in the case of QG. I will save the details for the second caveat following Case 5 but very briefly, the spin-networks of LQG are not even locate-able since, in general, there is no spacetime for them to be located within.

Recalling $\S 4.1$, when spin-networks take the form of weave-states, the world according to this theory comes to look as though there is geometry which can then be used to define spatial location. If there are physical spin-networks, then one 
might object to the preceding claims by noting that there is a sense in which the networks are in spacetime. Since the nodes and links of weave-state spin-networks are responsible for producing Reimannian volumes and areas, one might claim that the network is located there within those produced spatial structures. There are a few problems with this suggestion. The most relevant problem being that the great majority of spin-network states are not weave-states and, therefore, have no chance of representing structures in spacetime nor structures locate-able within spacetime. This fact is important for no matter the difference between abstract and concrete objects, that difference should not be contingent upon the form taken by the quantum geometry of LQG. Allow me to explain. If there is a metaphysical distinction between abstract and concrete objects, this distinction (I assume) is supposed to hold no matter which form the physical stuff of the universe takes. We all agree that the if $\pi$ and fish are metaphysically different sorts of things this difference, should not be dependent upon such contingent facts as how swiftly the fish is swimming. Similarly, that spin-networks are concrete should not be dependent upon their taking the form of some weave-state.

Case 2: Similar to the previous case, it has been widely thought that the abstractconcrete distinction rests on causal efficacy. Fish and red robins are causally efficacious and numbers are not. This causal distinction also fails in some versions of QG. If LQG is true, for instance, causes as standardly construed are not present in the theory. The physical world, according to LQG, does not have a fundamental metrical structure. Without a metrical structure, there are good reasons to doubt that the world includes much by way of change or variation at the fundamental level. And, without change, in what sense are there causes? This is not to suggest that there are no causes; however, if a cause is at least partially constituted by its effect, then were geometric properties to disappear so too would any cause whose effect is geometric in nature.

For example, let us assume that yesterday there was a large rectangular window near a book case and that today the window lies in a pile of irregularly shaped small glass shards much further from the book case. Standardly, the change in the state of the window would signal some sort of cause, a baseball perhaps, responsible for the change. However, in a world which lacks the metric properties modeled by $G$, since there are no lengths, there are no rectangles, no irregular shapes, no small, no near, no far. Without the physical geometry encoded by the metric field $G$, the sense of change which the world includes is incredibly reduced. Consequently, the kind and number of causes are also reduced (Norton, 2015a). If one were inclined to save this account of the abstract-concrete distinction, the what we would need is a family of causes some of which are possessed by fish and red robins and whose effects are not 
dependent on geometry. If I were to defend the distinction in light of QG, this is the avenue I would attempt.

There is a sense in which some of these results hold for ST as well; though, in the case of LQG, there is an additional problem. The theory notoriously suffers from the "problem of time." This problem seems to entail that, as currently formulated, there are no dynamics in the theory. In a bit more detail: what is called the Hamiltonian constraint requires that the quantum geometric states of the theory not evolve as the coordinate $t$ evolves. If evolution means change over a temporal dimension, then, according to DeWitt, "the quantum theory can never yield anything but a static picture of the world" (1967, p.1119). Since the world is static according to LQG, we ought to wonder what sorts of causes exist in a world where nothing happens? This is not to say that causes are impossible in such a world. A frozen world is compatible with a God who causes the existence of the same world, moment by moment. Even if there are some esoteric species of causation in the world, our concern remains. The world of LQG does not include the robust and varied kinds of causes (as traditionally understood) which are required for the proposed distinction between abstract and concrete objects. On the present account, what distinguishes the red robin from redness is that the robins eat worms whereas redness does not. If LQG is true, neither the robin nor redness eats any worms.

Most modern researches in LQG believe that the theory contains dynamics somehow, somewhere. The literature and the work done on this topic is quite extensive. For instance see (Kuchar, 1992) and (Isham, 1992) or more recently (Anderson, 2012) for overviews of some different approaches for locating dynamics in LQG. This literature notwithstanding, my overall point remains: any metaphysician who is committed to grounding the abstract-concrete distinction in causation ought to pay attention to how or if the problem of time gets resolved in LQG. It seems likely that whatever solution we find will require a fundamental modification in our understanding of time and, with it, our understanding of causation.

Case 3: According to Frege, an object is abstract if and only if it is both nonmental and non-sensible (1884). The fundamental objects of QG, spin-networks in LQG and strings in ST, are non-mental to the extent that even if there were no minds, they would still exist. Moreover, both spin-networks and strings are manifest only at energy scales which far outstrip our biological sensory equipment. Strictly speaking, with respect to human sensation, QG posits the existence of objects which are non-sensible. Perhaps Frege intended his definition to be interpreted less literally than this. Perhaps an object is non-sensible, in Frege's sense, when it is not possible for any creature in existence today or future product of evolution, to sense. Or perhaps, 'non-sensible' is meant to apply to all logically possible kinds of humans 
and their varied ways of sensing. If we take the modality in Frege's definition too broadly, as in the latter case, we run into a different kind of trouble. First note that there is no logical contradiction in a state of affairs which includes humans who sense $\pi .^{25}$ If we first assume, contrary to the preceding paragraph, that unlike concrete objects, abstract objects are non-causal, then a logical contradiction could be derived from the sentence "a human can sense $\pi$ where $\pi$ is an abstract object and sensing requires causal contact." However since the nature of abstract objects is whats under investigation, we cannot presuppose an answer to that question. Carrying on then, since there is a logically possible world with humans who can sense $\pi, \pi$ is sensible and therefore not abstract. Interpreting Frege's definition too broadly, as we have just done, and numbers fail to be abstract.

One might fear that I was too quick here and perhaps it is not logically possible for humans to sense $\pi$. If so, then there must be some logical contradiction in saying so. Taking a clue from Quine (1951), maybe the contradiction arises once we substitute words, salva veritate, for their meaning, or for a synonym? Consider that what it means to be a human is that numbers are non-sensible for them. On this proposal, in order that Ralph be human it must be the case that Ralph cannot sense numbers. If this were the meaning of 'human' then 'a human can sense a number' would be logically equivalent to 'a being who cannot sense numbers can sense a number'. Ergo, the contradiction we were looking for. Admittedly, this proposal allows us to interpret Frege broadly and keep spin-networks concrete and numbers abstract; though, it will result in other trouble.

Since Frege argues (1884) that mathematical objects are non-mental, numbers are abstract if and only if they are non-sensible. However, on the current proposal, Ralph is a human if and only if numbers are non-sensible. Thus, Ralph is a human if and only if numbers are abstract. Consequently, the meaning of being human is that numbers are abstract. If that seems odd, alternatively we might conclude that 'Ralph is a human' is logically equivalent to 'numbers are abstract'. In either case, this surely is not what Frege intended by his definition of 'abstract' nor what we intend by 'human.' We, with Frege, seem to be in a pickle. If we interpret the modality in 'non-sensible' literally, then spin-networks and strings are abstract. If we interpret the modality inherent in Frege's definition too broadly, then numbers fail to be abstract. I do not intend to argue for some particular modal interpretation of 'non-sensible' but aim merely to show that QG makes trouble for Frege's trichotomy of abstract, mental, and sensible objects.

${ }^{25}$ In fact, the actual world may contain such individuals. Those who suffer from synesthesia, arguably, sense mathematical objects. Interestingly, recent research has shown that the color sensation is not necessarily tied to concrete symbols (Gertner, 2013). 
Case 4: According to Donald C. Williams abstract objects, like concrete objects are spatiotemporal. There is no Platonic realm where abstract objects live, but rather abstract objects are particular things in the natural world. Williams denies that abstract objects should be equated with the "abstruse, the ethereal, the mental, the rational, the incorporeal, the ideally perfect, the non-temporal, the primordial or ultimate, the purely theoretical, the precariously speculative and visionary; or again with the... ." (1953, p.14) Abstract objects for Williams are merely abstractions from concrete things. Reality, according to Williams, is built out of abstract particulars (tropes) which, when compresent - co-located - in clusters, form concrete particulars. The concrete cat is "the collocation, or peculiar interpenetration, the unique congress in the same volume" (p.8) of very particular tropes including particular colors and shapes. What distinguishes abstract objects from concrete objects is grounded in spacetime regions or spatiotemporal comprescence. If spacetime fails to exist fundamentally, then so too does the distinction between abstract and concrete particulars. No tropes are spatiotemporally clustered according to QG since there is no space or spacetime, fundamentally, according to both ST and LQG. For similar reasons, Plato's distinction between universals and concrete particulars also fails to hold if QG is true.

Case 5: The following example is an amuse-bouche and not about the abstractconcrete distinction per se. According to Descartes, the primary attribute of body is extension. Consequently a substance cannot exist qua body if it lacks spatial extension whereas both God and minds can. If there are three kinds of substances: God, mind and body, then since there is no spatial extension in QG, there are no bodies. What then are tables, chair, and spin-networks? Presumably they are neither God nor thinking substances and must therefore be ideas in some mind, God's perhaps. It would seem that Berkeley knew more about the quantum world than he let on!

Two caveats are in order. First, many of the preceding accounts of the abstractconcrete distinction flounder for reasons independent of quantum theories of gravity. For instance, it is not only the strings of ST or the networks of LQG which cause problems for Frege's account. Since standard quantum particles are also non-sensible, on similar grounds, they too show Frege's account to be ill-suited to its task. Moreover, as already mentioned, quantum particles raise problems for the spatial-account of the abstract-concrete distinction. My argument here is not that LQG provides the only challenge to our metaphysics of abstract objects but that it provides $a$ challenge and that it would behoove anyone interested in doing metaphysics to keep these theories in mind.

Any metaphysical account of the abstract-concrete distinction which accommo- 
dates the challenge posed by quantum particles and yet fails to take into account the disappearance of spacetime under QG, will fail to be a physically informed metaphysics. In incubating a future metaphysics one cannot work to accommodate only some challenges while ignoring others. It does little good to patch only some of the holes in a sinking ship. Moreover, unlike other challenges to the abstract-concrete distinction, the challenge posed by quantum gravity is global. Because spacetime has a tenuous existence in quantum theories of gravity, these theories challenge $e v$ ery formulation of the distinction and not merely some particular formulation(s). For instance, though quantum particles threaten both the spacetime distinction as well as Frege's distinction, they leave the causal distinction unchallenged.

The second caveat is rather important. In $\S 4.1$ and 4.2 I mentioned that some interpret spacetime as being an emergent structure in both ST and LQG. The concern is that, if spacetime exists as a non-fundamental emergent structure, don't many of the preceding concerns fall away? For instance, while it might be admitted that the abstract-concrete distinction of Case 1 fails if spacetime does not exist, it might yet be maintained that this need not worry us if spacetime is emergent. According to this objection, my criticisms do not take seriously the possibility of emergentspacetime. Surely emergent spacetime, it will be argued, is sufficient to play the role that spacetime was to play? One version of this concern was already addressed as part of Case 1. Let us consider more generally what an account of the abstractconcrete distinction would say about the world if it utilized an emergent concept of spacetime rather than treating spacetime as fundamental. To be clear, an emergent spacetime only "exists", in a theory like LQG, at certain low-energies and only when the states of the theory happen to be weave-states.

It is true that at low energies, when using weakly powered tools such as human sensory organs, the fundamental quantum geometry, or "quantum spacetime", takes on the appearance of classical spacetime. This much is mostly uncontroversial in QG (qua LQG and ST). However, the current objection goes one step further and proposes that classical spacetime is an actual novem of reality at those low energies. When using our human sensory tools, quantum geometry fades out of existence and in its place a real classical geometric spacetime comes into existence - part and whole - just as it is in GR. While I am inclined to think that there are metaphysical incoherencies lurking about this suggestion, I am not interested in teasing them out. I am, however, interested in seeing whether or not emergent spacetime, as described here, is able to save the abstract-concrete distinction.

According to this proposal, the concreteness of concrete objects is somehow grounded in the existence, at low-energy scales, of a non-fundamental emergent spacetime structure. Perhaps it might be thought that concrete objects are just 
those things located in emergent spacetime. Or, perhaps, concrete objects are just those things which are causal at the energy scales at which a non-fundamental, emergent spacetime exists. I hope that one can start to see that something odd is afoot. There are at least three families of concerns.

Firstly, according to this proposal, emergent spacetime exists only at low energies and yet every energy scale is present at all moments. What should we say at energy scales higher than that designated for the emergent structures? When using high powered tools to probe the geometry at the Planck scale, when we move "out" of the low energy regime, are we supposed to conclude that since there is no fundamental or emergent spacetime at those energies that there is also no abstract-concrete distinction? Do fish and the number $\pi$ somehow stop being distinct kinds of objects at certain energy scales? Recall again that all energy scales exist at all moments of time which means that for any moment there is an energy at which emergent spacetime exists and an energy at which it does not. Thus, if we tie our metaphysics of abstract objects to emergent spacetime, then for every moment of time, there is an energy at which the abstract-concrete distinction holds and another at which it does not. I take such a conclusion to be rather unfortunate if not a reductio against the position.

Secondly, what is this talk of energy scales? What does the metaphysical structure of reality know about the "low" energy scales at which humans will begin to experience the quantum reality of LQG and ST as resembling the spacetime of GR? Is there some physical fine-tuning sensitive to human experience which causes a real classical spacetime to emerge exactly at those scales relevant to human sensory experiences? Is there some metaphysical fine-tuning sensitive to human experience which forces a metaphysical distinction between fish and $\pi$ just at those energies relevant for humans to experience quantum geometry as emergent-spacetime? It seems to me that any self-respecting abstract object should be abstract simpliciter at all energy scales, let alone at those energy scales preferred by humans.

Thirdly, as discussed in Case 1, most all spin-networks don't, in fact, describe a world with an emergent spacetime structure. There are many states in LQG and the vast majority of them are not weave-states. ${ }^{26}$ Should we conclude that when the states of LQG evolve into those very special weave-states that there is a concreteabstract distinction, and when they evolve out of those states that the distinction falls apart? Do we think that there being a metaphysical distinction between fish and the number $\pi$ is contingent, moment by moment, upon which quantum-geometric state the world is in? If LQG is true, then there are physically possible LQG-worlds in which the quantum geometry never takes the form of a weave-state. For all times, these worlds never include an emergent spacetime. In these worlds, using the proposal

\footnotetext{
${ }^{26}$ There are uncountably many states of LQG and only countably many of these are weave-states.
} 
under consideration, it would be false that there is an abstract-concrete distinction. It seems to me that if there is a distinction between abstract and concrete objects the distinction's existence is not contingent upon humans or the physical state of the universe, in which case, emergent-spacetime is of no use in saving the distinction. This is not to say that there are no means of saving the distinction in terms of emergent spacetime, but that such accounts will need to perform some complicated philosophical gymnastics.

\section{$5 \quad$ In Summary}

Despite our best efforts, many of our putatively metaphysical commitments are tied up with our theories about the physical world. We saw that this was the case in Copernican revolution and again in the case of modern theories of cosmology and gravity. Given this interdependence, I have argued that no matter our opinion on the relation of physics to metaphysics, we should take care not to hold too tightly onto what it is that we take to be immutable and independent of our physics. Moreover, I have explicated some features of LQG and ST and argued that if these theories are true that some aspects of our current metaphysics might not be true. The surface level lesson is that there is a conflict between QG and the abstract-concrete distinction. The more significant lesson is that QG is able to challenge deeply ingrained metaphysical presuppositions and is therefore a powerful tool in incubating a future metaphysics.

Even if the theories of QG are false or the received interpretations of them are, what they reveal is the potential contingency of our background metaphysics. Copernicus showed that the actual world does not include quintessential heavenly natures which eternally orbit the universe. Strictly speaking, Copernican cosmology is false and yet we are grateful for its lessons. So too, perhaps the actual world is not described by any quantum theory of gravity, but this does not mean that we cannot learn lessons from it about the actual structure of the world. Even if false, quantum theories of gravity show us how some aspects of our metaphysics might come into conflict with our future physics. Regarding those doctrines which reside at the center of our web of concepts, or which play a robust role in being, or in providing, truth-makers for important propositions, we often slip into a dogmatic attitude about them. The field of QG provide a heuristic for combating this metaphysical dogmatism. Seeing that the truth of QG might show our favorite metaphysics to be

false, we are forced to acknowledge that this piece of metaphysics might not actually be immune from revision. 


\section{Acknowledgments}

I would like to thank Max Kistler and Melissa Norton for their comments on earlier drafts of this paper as well as Courtney Fugate for his helpful guidance. I would also like to thank the participants of the New Trends in the Metaphysics of Science (2015) conference for their comments and conversation which inspired the form of this paper. Lastly, I would like to thank the anonymous referees (one tireless referee in particular) for providing very useful and thorough comments. 


\section{References}

[1] Anderson, E.: Problem of time in quantum gravity. Annalen der Physik 524(12), 757-86 (2012)

[2] Aristotle: Metaphysics. classics.mit.edu

[3] Aristotle: On interpretation. classics.mit.edu

[4] Ayer, A.: Language, Truth and Logic. Dover Publications (1936/1952)

[5] Baker, D.: Does string theory posit extended simples? Philosophers' Imprint 16(18) (2016)

[6] Belot, G., Earman, J.: Pre-socratic quantum gravity. In: N. Huggett, C. Callender (eds.) Physics Meets Philosophy at the Planck Scale, chap. 10, p. 213. Cambridge University Press (2001)

[7] Birkhoff, G., von Neumann, J.: The logic of quantum mechanics. Annals of Mathematics 37(4), 823-843 (1936)

[8] Dawid, R.: Scientific realism in the age of string theory. Physics and Philosophy 011 (2007)

[9] DeWitt, B.: Quantum theory of gravity. i. the canonical theory. Physical Review 160(5), 1113 (1967)

[10] Donald, W.C.: On the elements of being: I. The Review of Metaphysics 7(1), 3-18 (1953)

[11] Earman, J.: Bangs, Crunches, Whimpers, and Shrieks: Singularities and Acausalities in Relativistic Spacetimes. Oxford University Press (1995)

[12] Earman, J.: Thoroughly modern mctaggart: or, what mctaggart would have said if he had read the general theory of relativity. Philosophers' Imprint 2(3), $1(2002)$

[13] van Fraassen, B.: The Scientific Image. Oxford University Press (1980)

[14] Frege, G.: The Foundations of Arithmetic. Oxford: Blackwell (1884/1959)

[15] Gertner, L., Arend, I., Henik, A.: Numerical synesthesia is more than just a symbol-induced phenomenon. Frontiers in Psychology 4(860) (2013) 
[16] Huggett, N.: Target space ? space. Studies in the History and Philosophy of Modern Physics (Forthcoming)

[17] Huggett, N., Norton, J.: Weak discernibility for quanta, the right way. British Journal for the Philosophy of Science 65(1) (2013)

[18] Huggett, N., Vistarini, T.: Deriving general relativity from string theory. Philosophy of Science 82(5), 1163-1174 (2015)

[19] Huggett, N., Wüthrich, C.: Emergent spacetime and empirical (in) coherence. Studies in History and Philosophy of Modern Physics 44(3), 276-285 (2013)

[20] Isham, C.: Canonical quantum gravity and the problem of time,. Salamanca 1992, Proceedings, Integrable Systems, Quantum Groups, and Quantum Field Theories 409, 157 (1992)

[21] Kant, I.: Inaugural dissertation of 1770. Columbia College (1770/1894)

[22] Kant, I.: Critique of pure reason. Cambridge University Press (1998)

[23] Kuchar, K.: Time and interpretations of quantum gravity. In: G. Kunstatter, D. Vincent, W. J. (eds.) 4th Canadian Conference on General Relativity and Relativistic Astrophysics (1992)

[24] Kuhn, T.: The Copernican Revolution. Harvard University Press (1957/2003)

[25] Ladyman, J., Ross, D.: Every Thing Must Go. Oxford University Press (2007)

[26] Ladyman, J., Ross, D.: Scientific Metaphysics. Oxford University Press (2013)

[27] Lam, V.: Quantum structure and spacetime. In: T. Bigaj, C. Wüthrich (eds.) Metaphysics in Contemporary Physics. Rodopi (2016)

[28] Lam, V., Esfeld, M.: A dilemma for the emergence of spacetime in quantum gravity. Studies in History and Philosophy of Modern Physics: Part B 44(3), 286-293 (2013)

[29] Leibniz, G.: Discourse On Metaphysics Correspondence with Arnauld and Modology. 1873 Press (1902)

[30] Lukasiewicz, J.: On three-valued logic. In: L. Borkowski (ed.) Selected works by Jan Lukasiewicz. North-Holland Pub. Co. (1970) 
[31] Maddy, P.: Realism In Mathematics. Oxford University Press (1990)

[32] Matsubara, K.: Realism, underdetermination and string theory dualities. Synthese 190(3), 471-489 (2013)

[33] Maudlin, T.: Thoroughly muddled mctaggart or how to abuse gauge freedom to generate metaphysical monstrosities. Philosophers' Imprint 2(4) (2002)

[34] Muller, F., Saunders, S.: Discerning fermions. The British Journal of the Philosophy of Science 3(58), 499-548 (2008)

[35] Norton, J.: Quantum ontology. Ph.D. thesis, University of Illinois at Chicago (2015)

[36] Norton, J.: Weak discernibility and relations between quanta. Philosophy of Science 82(5), 1188-1199 (2015)

[37] Oriti, D.: Disappearance and emergence of space and time in quantum gravity. Studies in History and Philosophy of Modern Physics 46, 186-199 (2014)

[38] Pooley, O.: A hole revolution, or are we back where we started? History and Philosophy of Science B 37(2), 372-80 (2006)

[39] Quine, W.V.: Two dogmas of empiricism. The Philosophical Review 60, 20-43 (1951)

[40] Quine, W.V.: Epistemology naturalized. In: Ontological Relativity and Other Essays. Columbia University Press (1969)

[41] Rickles, D.: A new spin on the hole argument. Studies in History and Philosophy of Science B 36(3), 415-34 (2005)

[42] Rickles, D.: Bringing the hole argument back in the loop: A response to pooley. Studies in History and Philosophy of Science B 37(2), 381-87 (2006)

[43] Rickles, D.: A philosopher looks at string dualities. Studies in History and Philosophy of Science Part B 42(1), 54-67 (2011)

[44] Rickles, D.: Ads/cft duality and the emergence of spacetime. Studies in History and Philosophy of Modern Physics 44(3), 312-320 (2013)

[45] Rickles, D.: Mirror symmetry and other miracles in superstring theory. Foundations of Physics 43(1) (2013) 
[46] Rovelli, C.: Quantum Gravity. Cambridge University Press (2004)

[47] Smith, N.K.: A Commentary to Kant's 'Critique of Pure Reason'. Palgrave Macmillian (1918/2003)

[48] Teh, N.: Holography and emergence. Studies in History and Philosophy of Modern Physics 44(3), 300-311 (2013)

[49] Wüthrich, C.: Raiders of the lost spacetime. In: D. Lehmkuhl (ed.) Towards a Theory of Spacetime Theories. Birkhäuser Basel (2017 (forthcoming)) 